\title{
Application of Generalized Non-Local Quantum Hydrodynamics to the Calculation of the Charge Inner Structures for Proton and Electron
}

\author{
Boris V. Alexeev \\ Moscow University of Fine Chemical Technologies Prospekt Vernadskogo, Moscow, Russia \\ Email: Boris.Vlad.Alexeev@gmail.com
}

Received October 2, 2012; revised November 3, 2012; accepted November 13, 2012

\begin{abstract}
The proton and electron charge inner structures are considered in the frame of the non-local quantum hydrodynamics based on the non-local physical description. From calculations follow that proton and electron can be considered like charged balls (shortly CB model) which charges are concentrated mainly in the shell of these balls. The proton-electron collision in the frame of CB-model should be considered as collision of two resonators. In this case can be explained a number of character collisional features depending on the initial and final electron energies and the scattering angles.
\end{abstract}

Keywords: Foundations of the Theory of Transport Processes; The Theory of Solitons; Generalized Hydrodynamic Equations; Foundations of Quantum Mechanics

\section{Introduction}

\section{About the basic principles of the Generalized Quan- tum Hydrodynamics (GQH).}

I begin with the short reminding of basic principles of $\mathrm{GQH}$ created in particular in [1-8]. As it is shown the theory of transport processes (including quantum mechanics) can be considered in the frame of the unified theory based on the non-local physical description. In particular the generalized hydrodynamic equations represent an effective tool for solving problems in the very vast area of physical problems. For simplicity in introduction, we will consider fundamental methodic aspects from the qualitative standpoint of view avoiding excessively cumbersome formulas. A rigorous description is found, for example, in the monograph [6].

Let us consider the transport processes in open dissipative systems and ideas of following transformation of generalized hydrodynamic description in quantum hydrodynamics which can be applied to the individual particle.

The kinetic description is inevitably related to the system diagnostics. Such an element of diagnostics in the case of theoretical description in physical kinetics is the concept of the physically infinitely small volume. The correlation between theoretical description and system diagnostics is well-known in physics. Suffice it to recall the part played by test charge in electrostatics or by test circuit in the physics of magnetic phenomena. The tradi- tional definition of PhSV contains the statement to the effect that the PhSV contains a sufficient number of particles for introducing a statistical description; however, at the same time, the PhSV is much smaller than the volume $V$ of the physical system under consideration; in a first approximation, this leads to local approach in investigating of the transport processes. It is assumed in classical hydrodynamics that local thermodynamic equilibrium is first established within the PhSV, and only after that the transition occurs to global thermodynamic equilibrium if it is at all possible for the system under study. Let us consider the hydrodynamic description in more detail from this point of view. Assume that we have two neighboring physically infinitely small volumes $\mathbf{P h S V}_{\mathbf{1}}$ and $\mathbf{P h S V}_{2}$ in a non-equilibrium system. The one-particle distribution function (DF) $f_{s m, 1}\left(\boldsymbol{r}_{1}, \boldsymbol{v}, t\right)$ corresponds to the volume $\mathbf{P h S V}_{\mathbf{1}}$, and the function $f_{s m, 2}\left(\boldsymbol{r}_{2}, \boldsymbol{v}, t\right)$ - to the volume $\mathbf{P h S V}_{2}$. It is assumed in a first approximation that $f_{s m, 1}\left(\boldsymbol{r}_{1}, \boldsymbol{v}, t\right)$ does not vary within $\mathbf{P h S V}_{\mathbf{1}}$, same as $f_{s m, 2}\left(\boldsymbol{r}_{2}, \boldsymbol{v}, t\right)$ does not vary within the neighboring volume $\mathbf{P h S V}_{2}$. It is this assumption of locality that is implicitly contained in the Boltzmann equation (BE). However, the assumption is too crude. Indeed, a particle near the boundary between two volumes, which experienced the last collision in $\mathbf{P h S V}_{\mathbf{1}}$ and moves toward $\mathbf{P h S V}_{2}$, introduces information about the $f_{s m, 1}\left(\boldsymbol{r}_{1}, \boldsymbol{v}, t\right)$ into the neighboring volume $\mathbf{P h S V}_{2}$. Similarly, a particle near the boundary between two volumes, which experienced the last collision in $\mathbf{P h S V}_{2}$ 
and moves toward $\mathbf{P h S V}_{\mathbf{1}}$, introduces information about the DF $f_{s m, 2}\left(\boldsymbol{r}_{2}, \boldsymbol{v}, t\right)$ into the neighboring volume $\mathbf{P h S V}_{\mathbf{1}}$. The relaxation over translational degrees of freedom of particles of like masses occurs during several collisions. As a result, "Knudsen layers" are formed on the boundary between neighboring physically infinitely small volumes, the characteristic dimension of which is of the order of the path length. Therefore, a correction must be introduced into the DF in the PhSV, which is proportional to the mean time between collisions and to the substantive derivative of the DF being measured (rigorous derivation is given in [6]). Let a particle of finite radius be characterized as before by the position $\boldsymbol{r}$ at the instant of time $t$ of its center of mass moving at velocity $\boldsymbol{v}$. Then, the situation is possible where, at some instant of time $t$, the particle is located on the interface between two volumes. In so doing, the lead effect is possible (say, for $\mathbf{P h S V}_{2}$ ), when the center of mass of particle moving to the neighboring volume $\mathbf{P h S V}_{2}$ is still in $\mathbf{P h S V}_{\mathbf{1}}$. However, the delay effect takes place as well, when the center of mass of particle moving to the neighboring volume (say, $\mathbf{P h S V} \mathbf{V}_{2}$ ) is already located in $\mathbf{P h S V}_{2}$ but a part of the particle still belongs to PhSV $_{1}$.

Moreover, even the point-like particles (starting after the last collision near the boundary between two mentioned volumes) can change the distribution functions in the neighboring volume. The adjusting of the particles dynamic characteristics for translational degrees of freedom takes several collisions. As result, we have in the definite sense "the Knudsen layer" between these volumes. This fact unavoidably leads to fluctuations in mass and hence in other hydrodynamic quantities. Existence of such "Knudsen layers" is not connected with the choice of space nets and fully defined by the reduced description for ensemble of particles of finite diameters in the conceptual frame of open physically small volumes, therefore-with the chosen method of measurement. This entire complex of effects defines non-local effects in space and time.

The physically infinitely small volume (PhSV) is an open thermodynamic system for any division of macroscopic system by a set of PhSVs. But the Boltzmann equation (BE) [1,9-12]

$$
D f / D t=J^{B}
$$

where $J^{B}$ is the Boltzmann collision integral and $D / D t$ is a substantive derivative, fully ignores nonlocal effects and contains only the local collision integral $J^{B}$. The foregoing nonlocal effects are insignificant only in equilibrium systems, where the kinetic approach changes to methods of statistical mechanics.

This is what the difficulties of classical Boltzmann physical kinetics arise from. Also a weak point of the classical Boltzmann kinetic theory is the treatment of the dynamic properties of interacting particles. On the one hand, as follows from the so-called "physical" derivation of $\mathrm{BE}$, Boltzmann particles are regarded as material points; on the other hand, the collision integral in the $\mathrm{BE}$ leads to the emergence of collision cross sections.

Notice that the application of the above principles also leads to the modification of the system of Maxwell equations. While the traditional formulation of this system does not involve the continuity equation, its derivation explicitly employs the equation

$$
\frac{\partial \rho^{a}}{\partial t}+\frac{\partial}{\partial \boldsymbol{r}} \cdot \boldsymbol{j}^{a}=0
$$

where $\rho^{a}$ is the charge per unit volume, and $\boldsymbol{j}^{a}$ is the current density, both calculated without accounting for the fluctuations. As a result, the system of Maxwell equations written in the standard notation, namely

$$
\begin{aligned}
& \frac{\partial}{\partial \boldsymbol{r}} \cdot \boldsymbol{B}=0, \frac{\partial}{\partial \boldsymbol{r}} \cdot \boldsymbol{D}=\rho^{a}, \\
& \frac{\partial}{\partial \boldsymbol{r}} \cdot \boldsymbol{E}=-\frac{\partial \boldsymbol{B}}{\partial t}, \frac{\partial}{\partial \boldsymbol{r}} \cdot \boldsymbol{H}=\dot{j}^{a}+\frac{\partial \boldsymbol{D}}{\partial t}
\end{aligned}
$$

contains

$$
\rho^{a}=\rho-\rho^{f l}, \boldsymbol{j}^{a}=\boldsymbol{j}-\boldsymbol{j}^{f l} .
$$

The $\rho^{f l}, \dot{j}^{f l}$ fluctuations calculated using the generalized Boltzmann equation are given, for example, in Ref. $[2,4,6]$. The violation of Bell's inequalities [13] is found for local statistical theories, and the transition to non-local description is inevitable.

The rigorous approach to the derivation of the kinetic equation relative to one-particle $\mathrm{DF} f\left(K E_{f}\right)$ is based on employing the hierarchy of Bogoliubov equations. Generally speaking, the structure of $K E_{f}$ is as follows:

$$
\frac{D f}{D t}=J^{B}+J^{n l}
$$

where $J^{n l}$ is the non-local integral term. An approximation for the second collision integral is suggested by me in generalized Boltzmann physical kinetics,

$$
J^{n l}=\frac{D}{D t}\left(\tau \frac{D f}{D t}\right)
$$

Here, $\tau$ is non-local relaxation parameter, in the simplest case - the mean time between collisions of particles, which is related in a hydrodynamic approximation with dynamical viscosity $\mu$ and pressure $p$,

$$
\tau p=\Pi \mu,
$$

where the factor $\Pi$ is defined by the model of collision of particles: for neutral hard-sphere gas, $\Pi=0.8$ $[11,12]$. All of the known methods of the kinetic equation derivation relative to one-particle DF lead to approxima- 
tion (1.6), including the method of many scales, the method of correlation functions, and the iteration method.

In the general case, the parameter $\tau$ is the non-locality parameter; in quantum hydrodynamics, its magnitude is correlated with the "time-energy" uncertainty relation $[7,8]$.

Now we can turn our attention to the quantum hydrodynamic description of individual particles. The abstract of the classical Madelung's paper [14] contains only one phrase: "It is shown that the Schrödinger equation for one-electron problems can be transformed into the form of hydrodynamic equations". The following conclusion of principal significance can be done from the previous consideration $[7,8]$ :

1) Madelung's quantum hydrodynamics is equivalent to the Schrödinger equation (SE) and leads to description of the quantum particle evolution in the form of Euler equation and continuity equation. Quantum Euler equation contains additional potential of non-local origin which can be written for example in the Bohm form.

2) SE is consequence of the Liouville equation as result of the local approximation of non-local equations.

3) Generalized Boltzmann physical kinetics leads to the strict approximation of non-local effects in space and time and after the transmission to the local approximation leads to parameter $\tau$, which on the quantum level corresponds to the uncertainty principle "time-energy".

4) Generalized hydrodynamic equations (GHE) lead to SE as a deep particular case of the generalized Boltzmann physical kinetics and therefore of non-local hydrodynamics.

In principal GHE needn't in using of the "time-energy" uncertainty relation for estimation of the value of the non-locality parameter $\tau$. Moreover the "time-energy" uncertainty relation does not lead to the exact relations and from position of non-local physics is only the simplest estimation of the non-local effects. Really, let us consider two neighboring physically infinitely small volumes $\mathbf{P h S V}_{1}$ and $\mathbf{P h S V}_{2}$ in a non-equilibrium system. Obviously the time $\tau$ should tends to diminish with increasing of the velocities $u$ of particles invading in the nearest neighboring physically infinitely small volume $\left(\mathbf{P h S V}_{1}\right.$ or $\left.\mathbf{P h S V}_{2}\right)$ :

$$
\tau=H / u^{n} \text {. }
$$

But the value $\tau$ cannot depend on the velocity direction and naturally to tie $\tau$ with the particle kinetic energy, then

$$
\tau=H /\left(m u^{2}\right),
$$

where $H$ is a coefficient of proportionality, which reflects the state of physical system. In the simplest case $H$ is equal to Plank constant $\hbar$ and relation (1.8) be- comes compatible with the Heisenberg relation. Possible approximations of $\tau$-parameter in details in the monograph [6] are considered. But some remarks of the principal significance should be done.

It is known that Ehrenfest adiabatic theorem is one of the most important and widely studied theorems in Schrödinger quantum mechanics. It states that if we have a slowly changing Hamiltonian that depends on time, and the system is prepared in one of the instantaneous eigenstates of the Hamiltonian then the state of the system at any time is given by an the instantaneous eigenfunction of the Hamiltonian up to multiplicative phase factors [15-19]. Since the establishment of this theorem many fundamental results have been obtained, such as LandauZener transition [15,16], the Gell-Mann-Low theorem [17], Berry phase [18] and holonomy [19].

The adiabatic theory can be naturally incorporated in generalized quantum hydrodynamics based on local approximations of non-local terms. In the simplest case if $\Delta Q$ is the elementary heat quantity delivered for a system executing the transfer from one state (the corresponding time moment is $t_{i n}$ ) to the next one (the time moment $t_{e}$ ) then

$$
\Delta Q=\frac{1}{\tau} 2 \delta(\bar{T} \tau)
$$

where $\tau=t_{e}-t_{i n}$ and $\bar{T}$ is the average kinetic energy. For adiabatic case Ehrenfest supposes that

$$
2 \bar{T} \tau=\Omega_{1}, \Omega_{2}, \cdots
$$

where $\Omega_{1}, \Omega_{2}, \cdots$ are adiabatic invariants. Obviously for Plank's oscillator (compare with (1.9))

$$
2 \bar{T} \tau=n h .
$$

Conclusion: adiabatic theorem and consequences of this theory deliver the general quantization conditions for non-local quantum hydrodynamics.

\section{Generalized Quantum Hydrodynamic Equations}

Strict consideration leads to the following system of the generalized hydrodynamic equations (GHE) [6] written in the generalized Euler form:

Continuity equation for species $\alpha$ :

$$
\begin{aligned}
& \frac{\partial}{\partial t}\left\{\rho_{\alpha}-\tau_{\alpha}\left[\frac{\partial \rho_{\alpha}}{\partial t}+\frac{\partial}{\partial \boldsymbol{r}} \cdot\left(\rho_{\alpha} \boldsymbol{v}_{0}\right)\right]\right\} \\
& +\frac{\partial}{\partial \boldsymbol{r}} \cdot\left\{\rho_{\alpha} \boldsymbol{v}_{0}-\tau_{\alpha}\left[\frac{\partial}{\partial t}\left(\rho_{\alpha} \boldsymbol{v}_{0}\right)+\frac{\partial}{\partial \boldsymbol{r}} \cdot\left(\rho_{\alpha} \boldsymbol{v}_{0} \boldsymbol{v}_{0}\right)\right.\right. \\
& \left.\left.+\overrightarrow{\mathrm{I}} \cdot \frac{\partial p_{\alpha}}{\partial \boldsymbol{r}}-\rho_{\alpha} \boldsymbol{F}_{\alpha}^{(1)}-\frac{q_{\alpha}}{m_{\alpha}} \rho_{\alpha} \boldsymbol{v}_{0} \times \boldsymbol{B}\right]\right\}=R_{\alpha},
\end{aligned}
$$

and continuity equation for mixture 


$$
\begin{aligned}
& \frac{\partial}{\partial t}\left\{\rho-\sum_{\alpha} \tau_{\alpha}\left[\frac{\partial \rho_{\alpha}}{\partial t}+\frac{\partial}{\partial \boldsymbol{r}} \cdot\left(\rho_{\alpha} v_{0}\right)\right]\right\} \\
& +\frac{\partial}{\partial \boldsymbol{r}} \cdot\left\{\rho \boldsymbol{v}_{0}-\sum_{\alpha} \tau_{\alpha}\left[\frac{\partial}{\partial t}\left(\rho_{\alpha} \boldsymbol{v}_{0}\right)+\frac{\partial}{\partial \boldsymbol{r}} \cdot\left(\rho_{\alpha} \boldsymbol{v}_{0} \boldsymbol{v}_{0}\right)+\overrightarrow{\mathrm{I}} \cdot \frac{\partial p_{\alpha}}{\partial \boldsymbol{r}}-\rho_{\alpha} \boldsymbol{F}_{\alpha}^{(1)}-\frac{q_{\alpha}}{m_{\alpha}} \rho_{\alpha} \boldsymbol{v}_{0} \times \boldsymbol{B}\right]\right\}=0 .
\end{aligned}
$$

Momentum equation for species

$$
\begin{aligned}
& \frac{\partial}{\partial t}\left\{\rho_{\alpha} \boldsymbol{v}_{0}-\tau_{\alpha}\left[\frac{\partial}{\partial t}\left(\rho_{\alpha} \boldsymbol{v}_{0}\right)+\frac{\partial}{\partial \boldsymbol{r}} \cdot \rho_{\alpha} \boldsymbol{v}_{0} \boldsymbol{v}_{0}+\frac{\partial p_{\alpha}}{\partial \boldsymbol{r}}-\rho_{\alpha} \boldsymbol{F}_{\alpha}^{(1)}-\frac{q_{\alpha}}{m_{\alpha}} \rho_{\alpha} \boldsymbol{v}_{0} \times \boldsymbol{B}\right]\right\}-\boldsymbol{F}_{\alpha}^{(1)}\left[\rho_{\alpha}-\tau_{\alpha}\left(\frac{\partial \rho_{\alpha}}{\partial t}+\frac{\partial}{\partial \boldsymbol{r}}\left(\rho_{\alpha} \boldsymbol{v}_{0}\right)\right)\right] \\
& -\frac{q_{\alpha}}{m_{\alpha}}\left\{\rho_{\alpha} \boldsymbol{v}_{0}-\tau_{\alpha}\left[\frac{\partial}{\partial t}\left(\rho_{\alpha} \boldsymbol{v}_{0}\right)+\frac{\partial}{\partial \boldsymbol{r}} \cdot \rho_{\alpha} \boldsymbol{v}_{0} \boldsymbol{v}_{0}+\frac{\partial p_{\alpha}}{\partial \boldsymbol{r}}-\rho_{\alpha} \boldsymbol{F}_{\alpha}^{(1)}-\frac{q_{\alpha}}{m_{\alpha}} \rho_{\alpha} \boldsymbol{v}_{0} \times \boldsymbol{B}\right]\right\} \times \boldsymbol{B} \\
& +\frac{\partial}{\partial \boldsymbol{r}} \cdot\left\{\rho_{\alpha} \boldsymbol{v}_{0} \boldsymbol{v}_{0}+p_{\alpha} \overrightarrow{\mathrm{I}}-\tau_{\alpha}\left[\frac{\partial}{\partial t}\left(\rho_{\alpha} \boldsymbol{v}_{0} \boldsymbol{v}_{0}+p_{\alpha} \overrightarrow{\mathrm{I}}\right)+\frac{\partial}{\partial \boldsymbol{r}} \cdot \rho_{\alpha}\left(\boldsymbol{v}_{0} \boldsymbol{v}_{0}\right) \boldsymbol{v}_{0}+2 \overleftrightarrow{\mathrm{I}}\left(\frac{\partial}{\partial \boldsymbol{r}} \cdot\left(p_{\alpha} \boldsymbol{v}_{0}\right)\right)\right.\right. \\
& \left.\left.+\frac{\partial}{\partial \boldsymbol{r}} \cdot\left(\overleftrightarrow{\mathrm{I}}_{\alpha} \boldsymbol{v}_{0}\right)-\boldsymbol{F}_{\alpha}^{(1)} \rho_{\alpha} \boldsymbol{v}_{0}-\rho_{\alpha} \boldsymbol{v}_{0} \boldsymbol{F}_{\alpha}^{(1)}-\frac{q_{\alpha}}{m_{\alpha}} \rho_{\alpha}\left[\boldsymbol{v}_{0} \times \boldsymbol{B}\right] \boldsymbol{v}_{0}-\frac{q_{\alpha}}{m_{\alpha}} \rho_{\alpha} \boldsymbol{v}_{0}\left[\boldsymbol{v}_{0} \times \boldsymbol{B}\right]\right]\right\} \\
& =\int m_{\alpha} \boldsymbol{v}_{\alpha} J_{\alpha}^{s t, e l} \mathrm{~d} \boldsymbol{v}_{\alpha}+\int m_{\alpha} \boldsymbol{v}_{\alpha} J_{\alpha}^{\text {st,inel } \mathrm{d} \boldsymbol{v}_{\alpha} .}
\end{aligned}
$$

Generalized moment equation for mixture

$$
\begin{aligned}
& \frac{\partial}{\partial t}\left\{\rho \boldsymbol{v}_{0}-\sum_{\alpha} \tau_{\alpha}\left[\frac{\partial}{\partial t}\left(\rho_{\alpha} \boldsymbol{v}_{0}\right)+\frac{\partial}{\partial \boldsymbol{r}} \cdot \rho_{\alpha} \boldsymbol{v}_{0} \boldsymbol{v}_{0}+\frac{\partial p_{\alpha}}{\partial \boldsymbol{r}}-\rho_{\alpha} \boldsymbol{F}_{\alpha}^{(1)}-\frac{q_{\alpha}}{m_{\alpha}} \rho_{\alpha} \boldsymbol{v}_{0} \times \boldsymbol{B}\right]\right\}-\sum_{\alpha} \boldsymbol{F}_{\alpha}^{(1)}\left[\rho_{\alpha}-\tau_{\alpha}\left(\frac{\partial \rho_{\alpha}}{\partial t}+\frac{\partial}{\partial \boldsymbol{r}}\left(\rho_{\alpha} \boldsymbol{v}_{0}\right)\right)\right] \\
& -\sum_{\alpha} \frac{q_{\alpha}}{m_{\alpha}}\left\{\rho_{\alpha} \boldsymbol{v}_{0}-\tau_{\alpha}^{(0)}\left[\frac{\partial}{\partial t}\left(\rho_{\alpha} \boldsymbol{v}_{0}\right)+\frac{\partial}{\partial \boldsymbol{r}} \cdot \rho_{\alpha} \boldsymbol{v}_{0} \boldsymbol{v}_{0}+\frac{\partial p_{\alpha}}{\partial \boldsymbol{r}}-\rho_{\alpha} \boldsymbol{F}_{\alpha}^{(1)}-\frac{q_{\alpha}}{m_{\alpha}} \rho_{\alpha} \boldsymbol{v}_{0} \times \boldsymbol{B}\right]\right\} \times \boldsymbol{B} \\
& +\frac{\partial}{\partial \boldsymbol{r}} \cdot\left\{\rho \boldsymbol{v}_{0} \boldsymbol{v}_{0}+p \overrightarrow{\mathrm{I}}-\sum_{\alpha} \tau_{\alpha}\left[\frac{\partial}{\partial t}\left(\rho_{\alpha} \boldsymbol{v}_{0} \boldsymbol{v}_{0}+p_{\alpha} \overrightarrow{\mathrm{I}}\right)+\frac{\partial}{\partial \boldsymbol{r}} \cdot \rho_{\alpha}\left(\boldsymbol{v}_{0} \boldsymbol{v}_{0}\right) \boldsymbol{v}_{0}+2 \overrightarrow{\mathrm{I}}\left(\frac{\partial}{\partial \boldsymbol{r}} \cdot\left(p_{\alpha} \boldsymbol{v}_{0}\right)\right)\right.\right. \\
& \left.\left.+\frac{\partial}{\partial \boldsymbol{r}} \cdot\left(\overleftrightarrow{\mathrm{I}} p_{\alpha} \boldsymbol{v}_{0}\right)-\boldsymbol{F}_{\alpha}^{(1)} \rho_{\alpha} \boldsymbol{v}_{0}-\rho_{\alpha} \boldsymbol{v}_{0} \boldsymbol{F}_{\alpha}^{(1)}-\frac{q_{\alpha}}{m_{\alpha}} \rho_{\alpha}\left[\boldsymbol{v}_{0} \times \boldsymbol{B}\right] \boldsymbol{v}_{0}-\frac{q_{\alpha}}{m_{\alpha}} \rho_{\alpha} \boldsymbol{v}_{0}\left[\boldsymbol{v}_{0} \times \boldsymbol{B}\right]\right]\right\}=0
\end{aligned}
$$

Energy equation for component

$$
\begin{aligned}
& \frac{\partial}{\partial t}\left\{\frac{\rho_{\alpha} v_{0}^{2}}{2}+\frac{3}{2} p_{\alpha}+\varepsilon_{\alpha} n_{\alpha}-\tau_{\alpha}\left[\frac{\partial}{\partial t}\left(\frac{\rho_{\alpha} v_{0}^{2}}{2}+\frac{3}{2} p_{\alpha}+\varepsilon_{\alpha} n_{\alpha}\right)+\frac{\partial}{\partial \boldsymbol{r}} \cdot\left(\frac{1}{2} \rho_{\alpha} v_{0}^{2} \boldsymbol{v}_{0}+\frac{5}{2} p_{\alpha} \boldsymbol{v}_{0}+\varepsilon_{\alpha} n_{\alpha} \boldsymbol{v}_{0}\right)\right.\right. \\
& \left.\left.-\boldsymbol{F}_{\alpha}^{(1)} \cdot \rho_{\alpha} \boldsymbol{v}_{0}\right]\right\}+\frac{\partial}{\partial \boldsymbol{r}} \cdot\left\{\frac{1}{2} \rho_{\alpha} v_{0}^{2} \boldsymbol{v}_{0}+\frac{5}{2} p_{\alpha} \boldsymbol{v}_{0}+\varepsilon_{\alpha} n_{\alpha} \boldsymbol{v}_{0}-\tau_{\alpha}\left[\frac{\partial}{\partial t}\left(\frac{1}{2} \rho_{\alpha} v_{0}^{2} \boldsymbol{v}_{0}+\frac{5}{2} p_{\alpha} \boldsymbol{v}_{0}+\varepsilon_{\alpha} n_{\alpha} \boldsymbol{v}_{0}\right)\right.\right. \\
& +\frac{\partial}{\partial \boldsymbol{r}} \cdot\left(\frac{1}{2} \rho_{\alpha} v_{0}^{2} \boldsymbol{v}_{0} \boldsymbol{v}_{0}+\frac{7}{2} p_{\alpha} \boldsymbol{v}_{0} \boldsymbol{v}_{0}+\frac{1}{2} p_{\alpha} v_{0}^{2} \overrightarrow{\mathrm{I}}+\frac{5}{2} \frac{p_{\alpha}^{2}}{\rho_{\alpha}} \overrightarrow{\mathrm{I}}+\varepsilon_{\alpha} n_{\alpha} \boldsymbol{v}_{0} \boldsymbol{v}_{0}+\varepsilon_{\alpha} \frac{p_{\alpha}}{m_{\alpha}} \overrightarrow{\mathrm{I}}\right)-\rho_{\alpha} \boldsymbol{F}_{\alpha}^{(1)} \cdot \boldsymbol{v}_{0} \boldsymbol{v}_{0}-p_{\alpha} \boldsymbol{F}_{\alpha}^{(1)} \cdot \overrightarrow{\mathrm{I}} \\
& \left.\left.-\frac{1}{2} \rho_{\alpha} v_{0}^{2} \boldsymbol{F}_{\alpha}^{(1)}-\frac{3}{2} \boldsymbol{F}_{\alpha}^{(1)} p_{\alpha}-\frac{\rho_{\alpha} v_{0}^{2}}{2} \frac{q_{\alpha}}{m_{\alpha}}\left[\boldsymbol{v}_{0} \times \boldsymbol{B}\right]-\frac{5}{2} p_{\alpha} \frac{q_{\alpha}}{m_{\alpha}}\left[\boldsymbol{v}_{0} \times \boldsymbol{B}\right]-\varepsilon_{\alpha} n_{\alpha} \frac{q_{\alpha}}{m_{\alpha}}\left[\boldsymbol{v}_{0} \times \boldsymbol{B}\right]-\varepsilon_{\alpha} n_{\alpha} \boldsymbol{F}_{\alpha}^{(1)}\right]\right\} \\
& -\left\{\rho_{\alpha} \boldsymbol{F}_{\alpha}^{(1)} \cdot \boldsymbol{v}_{0}-\tau_{\alpha}\left[\boldsymbol{F}_{\alpha}^{(1)} \cdot\left(\frac{\partial}{\partial t}\left(\rho_{\alpha} \boldsymbol{v}_{0}\right)+\frac{\partial}{\partial \boldsymbol{r}} \cdot \rho_{\alpha} \boldsymbol{v}_{0} \boldsymbol{v}_{0}+\frac{\partial}{\partial \boldsymbol{r}} \cdot p_{\alpha} \overrightarrow{\mathrm{I}}-\rho_{\alpha} \boldsymbol{F}_{\alpha}^{(1)}-q_{\alpha} n_{\alpha}\left[\boldsymbol{v}_{0} \times \boldsymbol{B}\right]\right)\right]\right\} \\
& =\int\left(\frac{m_{\alpha} v_{\alpha}^{2}}{2}+\varepsilon_{\alpha}\right) J_{\alpha}^{s t, e l} \mathrm{~d} \boldsymbol{v}_{\alpha}+\int\left(\frac{m_{\alpha} v_{\alpha}^{2}}{2}+\varepsilon_{\alpha}\right) J_{\alpha}^{s t, \text { inel }} \mathrm{d} \boldsymbol{v}_{\alpha} .
\end{aligned}
$$


and after summation the generalized energy equation for mixture

$$
\begin{aligned}
& \frac{\partial}{\partial t}\left\{\frac{\rho v_{0}^{2}}{2}+\frac{3}{2} p+\sum_{\alpha} \varepsilon_{\alpha} n_{\alpha}-\sum_{\alpha} \tau_{\alpha}\left[\frac{\partial}{\partial t}\left(\frac{\rho_{\alpha} v_{0}^{2}}{2}+\frac{3}{2} p_{\alpha}+\varepsilon_{\alpha} n_{\alpha}\right)+\frac{\partial}{\partial \boldsymbol{r}} \cdot\left(\frac{1}{2} \rho_{\alpha} v_{0}^{2} \boldsymbol{v}_{0}+\frac{5}{2} p_{\alpha} \boldsymbol{v}_{0}+\varepsilon_{\alpha} n_{\alpha} \boldsymbol{v}_{0}\right)\right.\right. \\
& \left.\left.-\boldsymbol{F}_{\alpha}^{(1)} \cdot \rho_{\alpha} \boldsymbol{v}_{0}\right]\right\}+\frac{\partial}{\partial \boldsymbol{r}} \cdot\left\{\frac{1}{2} \rho v_{0}^{2} \boldsymbol{v}_{0}+\frac{5}{2} p \boldsymbol{v}_{0}+\boldsymbol{v}_{0} \sum_{\alpha} \varepsilon_{\alpha} n_{\alpha}-\sum_{\alpha} \tau_{\alpha}\left[\frac{\partial}{\partial t}\left(\frac{1}{2} \rho_{\alpha} v_{0}^{2} \boldsymbol{v}_{0}+\frac{5}{2} p_{\alpha} \boldsymbol{v}_{0}+\varepsilon_{\alpha} n_{\alpha} \boldsymbol{v}_{0}\right)\right.\right. \\
& +\frac{\partial}{\partial \boldsymbol{r}} \cdot\left(\frac{1}{2} \rho_{\alpha} v_{0}^{2} \boldsymbol{v}_{0} \boldsymbol{v}_{0}+\frac{7}{2} p_{\alpha} \boldsymbol{v}_{0} \boldsymbol{v}_{0}+\frac{1}{2} p_{\alpha} v_{0}^{2} \overrightarrow{\mathrm{I}}+\frac{5}{2} \frac{p_{\alpha}^{2}}{\rho_{\alpha}} \overrightarrow{\mathrm{I}}+\varepsilon_{\alpha} n_{\alpha} \boldsymbol{v}_{0} \boldsymbol{v}_{0}+\varepsilon_{\alpha} \frac{p_{\alpha}}{m_{\alpha}} \overrightarrow{\mathrm{I}}\right) \\
& -\rho_{\alpha} \boldsymbol{F}_{\alpha}^{(1)} \cdot \boldsymbol{v}_{0} \boldsymbol{v}_{0}-p_{\alpha} \boldsymbol{F}_{\alpha}^{(1)} \cdot \overrightarrow{\mathrm{I}}-\frac{1}{2} \rho_{\alpha} v_{0}^{2} \boldsymbol{F}_{\alpha}^{(1)}-\frac{3}{2} \boldsymbol{F}_{\alpha}^{(1)} p_{\alpha}-\frac{\rho_{\alpha} v_{0}^{2}}{2} \frac{q_{\alpha}}{m_{\alpha}}\left[\boldsymbol{v}_{0} \times \boldsymbol{B}\right] \\
& \left.\left.-\frac{5}{2} p_{\alpha} \frac{q_{\alpha}}{m_{\alpha}}\left[\boldsymbol{v}_{0} \times \boldsymbol{B}\right]-\varepsilon_{\alpha} n_{\alpha} \frac{q_{\alpha}}{m_{\alpha}}\left[\boldsymbol{v}_{0} \times \boldsymbol{F}\right]-\varepsilon_{\alpha} n_{\alpha} \boldsymbol{F}_{\alpha}^{(1)}\right]\right\}-\boldsymbol{v}_{0} \cdot \sum_{\alpha} \rho_{\alpha} \boldsymbol{F}_{\alpha}^{(1)} \\
& +\sum_{\alpha} \tau_{\alpha} \boldsymbol{F}_{\alpha}^{(1)} \cdot\left[\frac{\partial}{\partial t}\left(\rho_{\alpha} \boldsymbol{v}_{0}\right)+\frac{\partial}{\partial \boldsymbol{r}} \cdot \rho_{\alpha} \boldsymbol{v}_{0} \boldsymbol{v}_{0}+\frac{\partial}{\partial \boldsymbol{r}} \cdot p_{\alpha} \overrightarrow{\mathrm{I}}-\rho_{\alpha} \boldsymbol{F}_{\alpha}^{(1)}-q_{\alpha} n_{\alpha}\left[\boldsymbol{v}_{0} \times \boldsymbol{B}\right]\right]=0 .
\end{aligned}
$$

Here $\boldsymbol{F}_{\alpha}^{(1)}$ are the forces of the non-magnetic origin, $\boldsymbol{B}$-magnetic induction, $\overrightarrow{\mathrm{I}}$-unit tensor, $q_{\alpha}$-charge of the $\alpha$-component particle, $p_{\alpha}$-static pressure for $\alpha$-component, $\varepsilon_{\alpha}$-internal energy for the particles of $\alpha$-component, $\boldsymbol{v}_{0}$-hydrodynamic velocity for mixture. For calculations in the self-consistent electro-magnetic field the system of non-local Maxwell equations should be added (see (1.3)).

It is well known that basic Schrödinger equation (SE) of quantum mechanics firstly was introduced as a quantum mechanical postulate. The obvious next step should be done and was realized by E. Madelung in 1927-the derivation of special hydrodynamic form of SE after introduction wave function $\psi$ as

$$
\psi(x, y, z, t)=\alpha(x, y, z, t) e^{\mathrm{i} \beta(x, y, z, t)} .
$$

Using (2.7) and separating the real and imagine parts of SE one obtains

$$
\frac{\partial \alpha^{2}}{\partial t}+\frac{\partial}{\partial \boldsymbol{r}} \cdot\left(\frac{\alpha^{2} \hbar}{m} \frac{\partial \beta}{\partial \boldsymbol{r}}\right)=0,
$$

and Equation (2.8) immediately transforms in continuity equation if the identifications for density and velocity

$$
\begin{aligned}
& \rho=\alpha^{2}=\psi \psi^{*}, \\
& \boldsymbol{v}=\frac{\partial}{\partial \boldsymbol{r}}(\beta \hbar / m)
\end{aligned}
$$

introduce in Equation (2.8). Identification for velocity (2.10) is obvious because for $1 \mathrm{D}$ flow

$$
\begin{aligned}
v & =\frac{\partial}{\partial x}(\beta \hbar / m)=\frac{\hbar}{m} \frac{\partial}{\partial x}\left[-\frac{1}{\hbar}\left(E_{k} t-p x\right)\right], \\
& =\frac{1}{m} \frac{\partial}{\partial x}(p x)=v_{\phi}
\end{aligned}
$$

where $v_{\phi}$ is phase velocity. The existence of the condition (2.10) means that the corresponding flow has potential

$$
\varphi=\beta \hbar / m .
$$

As result two effective hydrodynamic equations take place:

$$
\begin{gathered}
\frac{\partial \rho}{\partial t}+\frac{\partial}{\partial \boldsymbol{r}} \cdot(\rho \boldsymbol{v})=0 \\
\frac{\partial \boldsymbol{v}}{\partial t}+\frac{1}{2} \frac{\partial}{\partial \boldsymbol{r}} v^{2}=-\frac{1}{m} \frac{\partial}{\partial \boldsymbol{r}}\left(U-\frac{\hbar^{2}}{2 m} \frac{\Delta \alpha}{\alpha}\right)
\end{gathered}
$$

But

$$
\frac{\Delta \alpha}{\alpha}=\frac{\Delta \alpha^{2}}{2 \alpha^{2}}-\frac{1}{\alpha^{2}}\left(\frac{\partial \alpha}{\partial \boldsymbol{r}}\right)^{2},
$$

and the relation (2.15) transforms (2.14) in particular case of the Euler motion equation

$$
\frac{\partial \boldsymbol{v}}{\partial t}+\left(\boldsymbol{v} \cdot \frac{\partial}{\partial \boldsymbol{r}}\right) \boldsymbol{v}=-\frac{1}{m} \frac{\partial}{\partial \boldsymbol{r}} U^{*}
$$

where introduced the efficient potential

$$
U^{*}=U-\frac{\hbar^{2}}{4 m \rho}\left[\Delta \rho-\frac{1}{2 \rho}\left(\frac{\partial \rho}{\partial \boldsymbol{r}}\right)^{2}\right] .
$$

Additive quantum part of potential can be written in the so called Bohm form

$$
\frac{\hbar^{2}}{2 m \sqrt{\rho}} \Delta \sqrt{\rho}=\frac{\hbar^{2}}{4 m \rho}\left[\Delta \rho-\frac{1}{2 \rho}\left(\frac{\partial \rho}{\partial \boldsymbol{r}}\right)^{2}\right] .
$$

Then 


$$
\begin{aligned}
U^{*} & =U+U_{q u}=U-\frac{\hbar^{2}}{2 m \sqrt{\rho}} \Delta \sqrt{\rho} \\
& =U-\frac{\hbar^{2}}{4 m \rho}\left[\Delta \rho-\frac{1}{2 \rho}\left(\frac{\partial \rho}{\partial \boldsymbol{r}}\right)^{2}\right] .
\end{aligned}
$$

Some remarks:

a) SE transforms in hydrodynamic form without additional assumptions. But numerical methods of hydrodynamics are very good developed. As result at the end of seventieth of the last century we realized the systematic calculation of quantum problems using quantum hydrodynamics (see for example $[1,20]$ ).

b) SE reduces to the system of continuity equation and particular case of the Euler equation with the additional potential proportional to $\hbar^{2}$. The physical sense and the origin of the Bohm potential are established later in $[7,8]$.

c) $\mathrm{SE}$ (obtained in the frame of the theory of classical complex variables) cannot contain the energy equation in principle. As result in many cases the palliative approach is used when for solution of dissipative quantum problems the classical hydrodynamics is used with insertion of additional Bohm potential in the system of hydrody- namic equations.

d) The system of the generalized quantum hydrodynamic equations contains energy equation written for unknown dependent value which can be specified as quantum pressure $p_{\alpha}$ of non-local origin.

In the following I intend to apply generalized non-local quantum hydrodynamic Equations (2.1)-(2.6) to investigation of the proton and electron internal charge structures.

\section{The Charge Internal Structures of Proton and Electron}

Let us consider a positive charged physical system placed in a bounded region of a space. Internal energy $\varepsilon_{\alpha}$ of this one species object and a possible influence of the magnetic field are not taken into account. The character linear scale of this region will be defined as result of the self-consistent solution of the generalized nonlocal quantum hydrodynamic Equations (2.1)-(2.6). Suppose also that the mentioned physical object for simplicity has the spherical form and the system (2.1)-(2.6) takes the form $[21,22]$ :

Continuity equation:

$$
\frac{\partial}{\partial t}\left\{\rho-\tau\left[\frac{\partial \rho}{\partial t}+\frac{1}{r^{2}} \frac{\partial\left(r^{2} \rho v_{0 r}\right)}{\partial r}\right]\right\}+\frac{1}{r^{2}} \frac{\partial}{\partial r}\left\{r^{2}\left\{\rho v_{0 r}-\tau\left[\frac{\partial}{\partial t}\left(\rho v_{0 r}\right)+\frac{1}{r^{2}} \frac{\partial\left(r^{2} \rho v_{0 r}^{2}\right)}{\partial r}+q \frac{\partial \phi}{\partial r}\right]\right\}\right\}-\frac{1}{r^{2}} \frac{\partial}{\partial r}\left(\tau r^{2} \frac{\partial p}{\partial r}\right)=0
$$

Momentum equation:

$$
\begin{aligned}
& \frac{\partial}{\partial t}\left\{\rho v_{0 r}-\tau\left[\frac{\partial}{\partial t}\left(\rho v_{0 r}\right)+\frac{1}{r^{2}} \frac{\partial\left(r^{2} \rho v_{0 r}^{2}\right)}{\partial r}+\frac{\partial p}{\partial r}+q \frac{\partial \phi}{\partial r}\right]\right\}+\frac{q}{\rho} \frac{\partial \phi}{\partial r}\left[\rho-\tau\left(\frac{\partial \rho}{\partial t}+\frac{1}{r^{2}} \frac{\partial\left(r^{2} \rho v_{0 r}\right)}{\partial r}\right)\right] \\
& +\frac{1}{r^{2}} \frac{\partial}{\partial r}\left\{r^{2}\left\{\rho v_{0 r}^{2}-\tau\left[\frac{\partial}{\partial t}\left(\rho v_{0 r}^{2}\right)+\frac{1}{r^{2}} \frac{\partial\left(r^{2} \rho v_{0 r}^{3}\right)}{\partial r}+2 q \frac{\partial \phi}{\partial r} v_{0 r}\right]\right\}\right\}+\frac{\partial p}{\partial r}-\frac{\partial}{\partial r}\left(\tau \frac{\partial p}{\partial t}\right) \\
& -2 \frac{\partial}{\partial r}\left(\frac{\tau}{r^{2}} \frac{\partial\left(r^{2} p v_{0 r}\right)}{\partial r}\right)-\frac{1}{r^{2}} \frac{\partial}{\partial r}\left(\tau r^{2} \frac{\partial\left(p v_{0 r}\right)}{\partial r}\right)=0 .
\end{aligned}
$$

Energy equation:

$$
\begin{aligned}
& \frac{\partial}{\partial t}\left\{\frac{1}{2} \rho v_{0 r}^{2}+\frac{3}{2} p-\tau\left[\frac{\partial}{\partial t}\left(\frac{1}{2} \rho v_{0 r}^{2}+\frac{3}{2} p\right)+\frac{1}{r^{2}} \frac{\partial}{\partial r}\left(r^{2} v_{0 r}\left(\frac{1}{2} \rho v_{0 r}^{2}+\frac{5}{2} p\right)\right)+q \frac{\partial \phi}{\partial r} v_{0 r}\right]\right\} \\
& +\frac{1}{r^{2}} \frac{\partial}{\partial r}\left\{r ^ { 2 } \left\{\left(\frac{1}{2} \rho v_{0 r}^{2}+\frac{5}{2} p\right) v_{0 r}-\tau\left[\frac { \partial } { \partial t } \left(\left(\frac{1}{2} \rho v_{0 r}^{2}+\frac{5}{2} p\right) v_{0 r}+\frac{1}{r^{2}} \frac{\partial}{\partial r}\left(r^{2}\left(\frac{1}{2} \rho v_{0 r}^{2}+\frac{7}{2} p\right) v_{0 r}^{2}\right)\right.\right.\right.\right. \\
& \left.\left.\left.+q \frac{\partial \varphi}{\partial r} v_{0 r}^{2}+\frac{q}{\rho} \frac{\partial \varphi}{\partial r}\left(\frac{1}{2} \rho v_{0 r}^{2}+\frac{3}{2} p\right)\right]\right\}\right\}-\left\{-q \frac{\partial \varphi}{\partial r} v_{0 r}-\tau\left[-\frac{q}{\rho} \frac{\partial \varphi}{\partial r}\left(\frac{\partial}{\partial t}\left(\rho v_{0 r}\right)+\frac{1}{r^{2}} \frac{\partial}{\partial r}\left(r^{2} \rho v_{0 r}^{2}\right)+\frac{\partial p}{\partial r}+q \frac{\partial \varphi}{\partial r}\right)\right]\right\} \\
& -\frac{1}{r^{2}} \frac{\partial}{\partial r}\left(\tau r^{2} \frac{\partial}{\partial r}\left(\frac{1}{2} p v_{0 r}^{2}+\frac{5}{2} \frac{p^{2}}{\rho}\right)\right)-\frac{1}{r^{2}} \frac{\partial}{\partial r}\left(r^{2} \tau p \frac{q}{\rho} \frac{\partial \varphi}{\partial r}\right)=0 .
\end{aligned}
$$


Moreover let us admit that stationary physical system is at the rest, namely $v_{0 r}=0$ and $\frac{\partial}{\partial t} \equiv 0$.

Is it possible to obtain the soliton type solution for this object under these stiff conditions? Let us show that the system (2.1)-(2.6) admits such kind of solutions. For mentioned case system (2.1)-(2.6) can be written as (see also (3.1)-(3.3):

Poisson equation:

$$
\frac{1}{r^{2}} \frac{\partial}{\partial r}\left(r^{2} \frac{\partial \varphi}{\partial r}\right)=-4 \pi q,
$$

where $\varphi$-scalar electric potential and $q$ is the positive charge (per the unit of volume) of the one species quantum object.

Continuity equation:

$$
\frac{\partial}{\partial r}\left\{\tau r^{2}\left(\frac{\partial p}{\partial r}+q \frac{\partial \varphi}{\partial r}\right)\right\}=0 .
$$

Momentum equation:

$$
\frac{\partial p}{\partial r}+q \frac{\partial \varphi}{\partial r}=0 .
$$

Equation (3.5) is satisfied for all parameter of non-locality if Equation (3.6) is fulfilled.

Energy equation takes the form:

$$
\begin{aligned}
& -\frac{1}{r^{2}} \frac{5}{2} \frac{\partial}{\partial r}\left\{r^{2} \tau p \frac{q}{\rho} \frac{\partial \varphi}{\partial r}\right\}+\tau \frac{q}{\rho} \frac{\partial \varphi}{\partial r}\left[\frac{\partial p}{\partial r}+q \frac{\partial \varphi}{\partial r}\right] \\
& -\frac{1}{r^{2}} \frac{5}{2} \frac{\partial}{\partial r}\left(\tau r^{2} \frac{\partial}{\partial r}\left(\frac{p^{2}}{\rho}\right)\right)=0
\end{aligned}
$$

or using(3.6)

$$
\frac{\partial}{\partial r}\left\{r^{2} \tau p \frac{q}{\rho} \frac{\partial \varphi}{\partial r}\right\}+\frac{\partial}{\partial r}\left(\tau r^{2} \frac{\partial}{\partial r}\left(\frac{p^{2}}{\rho}\right)\right)=0 .
$$

From (3.8) follows

$$
\tau p \frac{q}{\rho} r^{2} \frac{\partial \varphi}{\partial r}+\tau r^{2} \frac{\partial}{\partial r}\left(\frac{p^{2}}{\rho}\right)=C
$$

where $C$ is constant of integration. If the non-locality parameter $\tau$ does not depend explicitly on $r$ and the left side of Equation (3.9) turns into zero by $r=0$, then $C=0$. Equation (3.9) is written as

$$
p \frac{q}{\rho} \frac{\partial \varphi}{\partial r}+\frac{\partial}{\partial r}\left(\frac{p^{2}}{\rho}\right)=0
$$

or using (3.6) and the relation $\rho=m q / e$

$$
\frac{\partial}{\partial r}\left(\frac{p}{q}\right)=0,
$$

which leads to the solution with new constant of integra- tion $C$

$$
p=C q .
$$

From (3.6), (3.12) one obtains

$$
\ln \left(q / C_{1}\right)=-\varphi / C \text {. }
$$

with new constants of integration $C$ and $C_{1}$. Let us use these constants as scales, namely $C=\varphi_{0}, C_{1}=q_{0}$ denoting of dimensionless values by wave $\tilde{q}=q / q_{0}$, $\tilde{\varphi}=\varphi / \varphi_{0}$. Equation (3.4) transforms into dimensionless equation

$$
A \frac{\partial}{\partial \tilde{r}}\left(\tilde{r}^{2} \frac{\partial \tilde{\varphi}}{\partial \tilde{r}}\right)=-\tilde{r}^{2} \exp (-\tilde{\varphi}),
$$

where the form-factor is introduced

$$
A=\varphi_{0} /\left(4 \pi r_{0}^{2} q_{0}\right) .
$$

For other equations one obtains

$$
\begin{aligned}
& A \frac{\partial}{\partial \tilde{r}}\left(\tilde{r}^{2} \frac{1}{\tilde{q}} \frac{\partial \tilde{q}}{\partial \tilde{r}}\right)=\tilde{r}^{2} \tilde{q}, \\
& A \frac{\partial}{\partial \tilde{r}}\left(\tilde{r}^{2} \frac{1}{\tilde{p}} \frac{\partial \tilde{p}}{\partial \tilde{r}}\right)=\tilde{r}^{2} \tilde{p},
\end{aligned}
$$

where the scale for pressure is $p_{0}=C C_{1}=\varphi_{0} q_{0}$. Equations (3.16), (3.17) have the same dimensionless solutions. Definition (3.15) for dimensionless factor $A$ can be written as

$$
q_{0}=\varphi_{0} /\left(4 \pi r_{0}^{2} A\right) .
$$

It means that $C_{0, \text { cap }}=\left(4 \pi r_{0}^{2} A\right)^{-1}$ can be considered as the scale of proton capacity per unit of volume and for scale of volume $V_{0}=\frac{4}{3} \pi r_{0}^{3}$ the scale of proton capacity is equal to $r_{0}$ if $A=1 / 3$.

Figures 1 and 2 reflect the solutions of (3.14), (3.16) correspondingly for $A=1 / 3$, Maple notations are used

$$
\left(\mathrm{v}=\tilde{\varphi}, D(\mathrm{v})(t)=\frac{\partial \tilde{\varphi}}{\partial \tilde{r}}, q=\tilde{q}\right) .
$$

Cauchy conditions for these calculations:

$$
\begin{aligned}
& \mathrm{v}(0)=\tilde{\varphi}(0)=1, \\
& D(\mathrm{v})(0)=\frac{\partial \tilde{\varphi}}{\partial \tilde{r}}(0)=0 ; \\
& \tilde{q}(0)=\mathrm{e}^{-1}, \\
& D(q)(0)=\frac{\partial \tilde{q}}{\partial \tilde{r}}(0)=0 .
\end{aligned}
$$

From Figures 1 and 2 follow that solutions exist for this case in the domain less than $r / r_{0} \cong 3$.

From (3.16) follows that the proton charge $q_{p r}$ is equal to 


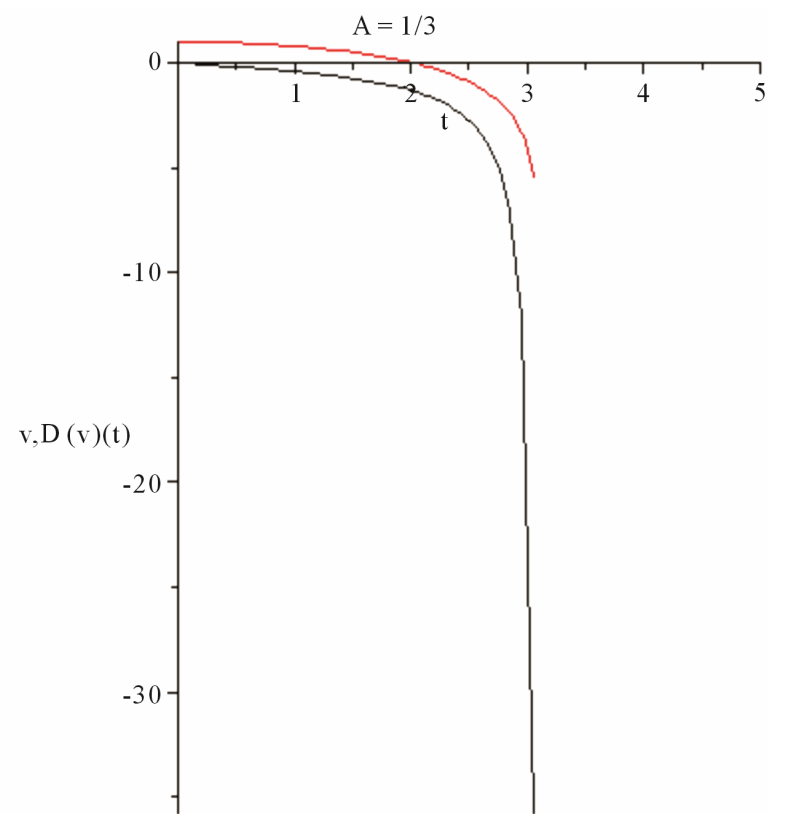

Figure 1. $\mathbf{v}=\tilde{\varphi}(\tilde{\boldsymbol{r}}), \quad D(\mathbf{v})(t)=\frac{\partial \tilde{\varphi}}{\partial \tilde{\boldsymbol{r}}}(\tilde{\boldsymbol{r}})$ for proton, red line $\mathbf{v}=\tilde{\varphi}(\tilde{\boldsymbol{r}})$.

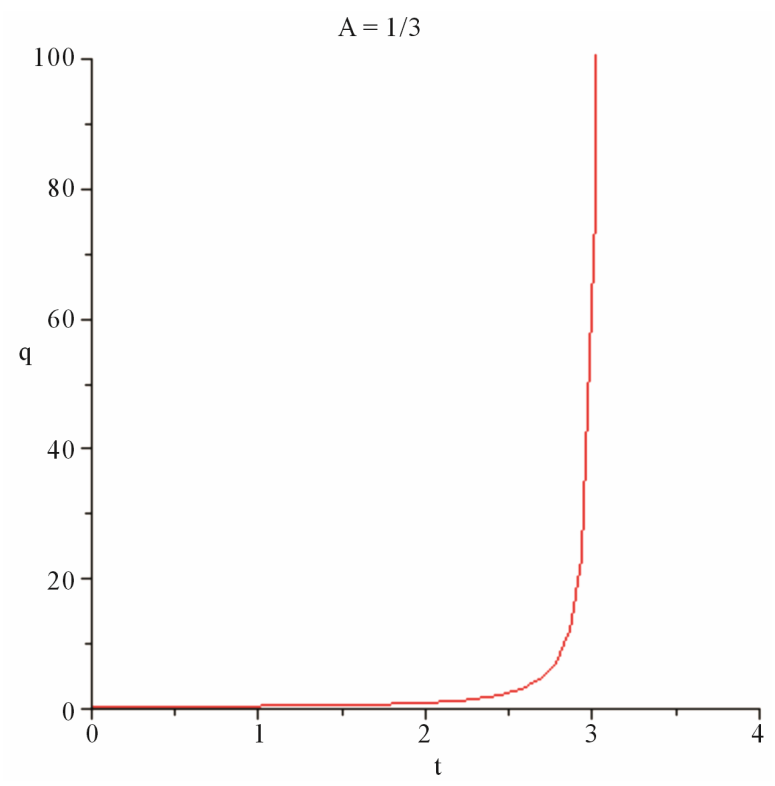

Figure 2. $q=\tilde{q}(\tilde{r})$ for proton.

$$
\begin{aligned}
q_{p r} & =\int_{0}^{r_{p r}} 4 \pi r^{2} q(r) \mathrm{d} r=4 \pi r_{0}^{3} q_{0} \int_{0}^{\tilde{r}_{p r}} \tilde{r}^{2} \tilde{q} \mathrm{~d} \tilde{r} \\
& =4 \pi r_{0}^{3} q_{0} A \int_{0}^{\tilde{r}_{p r}} \frac{\partial}{\partial \tilde{r}}\left(\tilde{r}^{2} \frac{1}{\tilde{q}} \frac{\partial \tilde{q}}{\partial \tilde{r}}\right) \mathrm{d} \tilde{r} \\
& =r_{0} \varphi_{0} \int_{0}^{\tilde{r}_{p r}} \frac{\partial}{\partial \tilde{r}}\left(\tilde{r}^{2} \frac{1}{\tilde{q}} \frac{\partial \tilde{q}}{\partial \tilde{r}}\right) \mathrm{d} \tilde{r}=r_{0} \varphi_{0} \tilde{r}_{p r}^{2}\left[\frac{\partial \ln \tilde{q}}{\partial \tilde{r}}\right]_{r=r_{p r}}
\end{aligned}
$$

or

$$
\tilde{q}_{p r}=\tilde{r}_{p r}^{2}\left[\frac{\partial \ln \tilde{q}}{\partial \tilde{r}}\right]_{r=r_{p r}}
$$

if $q_{p r, 0}=r_{0} \varphi_{0}$.

Relation (3.19) delivers the natural boundary condition for the external area of proton. For example if the restrictions $r_{0}=r_{p r}, \quad q_{0}=0.75 \frac{q_{p r}}{\pi r_{p r}^{3}}$ and $\varphi_{0}=r_{p r}^{2} q_{0}$ (therefore $\left.\varphi_{0}=\frac{3}{4 \pi} \frac{q_{p r}}{r_{p r}}\right)$ are introduced then

$$
\left[\frac{\partial \ln \tilde{q}}{\partial \tilde{r}}\right]_{r=r_{p r}}=\frac{q_{p r}}{r_{p r} \varphi_{0}}=\frac{4 \pi}{3}
$$

and in the vicinity of $r \sim r_{p r}$ we have

$$
\tilde{q}_{r=r_{p r}}=C\left[\exp \left(\frac{4 \pi}{3} \tilde{r}\right)\right]_{r=r_{p r}} .
$$

Obviously (3.21) can be written using the dimensional $\left(\mathrm{cm}^{-3}\right)$ form-factor $F_{q}$

$$
q\left(r=r_{p r}\right)=F_{q} q_{p r} .
$$

For the chosen scales

$$
A=\frac{\varphi_{0}}{4 \pi r_{p r}^{2} q_{0}}=\frac{1}{4 \pi} .
$$

As we see the choice of scales is the question of convenience by the interpretation of the experimental data and the corresponding choice of the Cauchy conditions.

Now we can apply the previous theory to the calculation of the internal electron structure. As before we intend to consider the electron at the rest placed in the self-consistent intrinsic electric field without an intrinsic magnetic field.

In our electron model, we no longer regard the electron as a point-like particle. Similar to the proton's electric charge, which has continuum distribution inside of the proton, we make the same basic assumptions based on the application of the non-local theory. On this step of investigation no reason to introduce the simplest model of electron spin like a spinning electrically charged ball or much more complicated theory which leads to the magnetic charge continuum distribution inside of the electron using the Dirac monopole speculations [23,24].

Obviously Poisson Equation (3.4) transforms into

$$
\frac{1}{r^{2}} \frac{\partial}{\partial r}\left(r^{2} \frac{\partial \varphi}{\partial r}\right)=4 \pi q
$$

where $\varphi$-scalar electric potential and $(-q)$ is the negative charge (per the unit of volume) of the one species quantum object. In other words $q$ is absolute charge 
density for electron. Two other Equations (3.16), (3.17) do not change the forms

$$
\begin{aligned}
& A \frac{\partial}{\partial \tilde{r}}\left(\tilde{r}^{2} \frac{1}{\tilde{q}} \frac{\partial \tilde{q}}{\partial \tilde{r}}\right)=\tilde{r}^{2} \tilde{q}, \\
& A \frac{\partial}{\partial \tilde{r}}\left(\tilde{r}^{2} \frac{1}{\tilde{p}} \frac{\partial \tilde{p}}{\partial \tilde{r}}\right)=\tilde{r}^{2} \tilde{p},
\end{aligned}
$$

where $A$ is the same dimensionless factor (3.15) but $\tilde{q}=\mathrm{e}^{\tilde{\rho}}$ and

$$
A \frac{\partial}{\partial \tilde{r}}\left(\tilde{r}^{2} \frac{\partial \tilde{\varphi}}{\partial \tilde{r}}\right)=\tilde{r}^{2} \exp (\tilde{\varphi}) .
$$

Figures 3 and $\mathbf{4}$ reflect the solutions of (3.27), (3.25) correspondingly for $A=1 / 3$, Maple notations are used $\left(\mathrm{v}=\tilde{\varphi} D(\mathrm{v})(t)=\frac{\partial \tilde{\varphi}}{\partial \tilde{r}}, q=\tilde{q}\right)$. Cauchy conditions for this calculations:

$$
\begin{aligned}
& \mathrm{v}(0)=\tilde{\varphi}(0)=1, \quad D(\mathrm{v})(0)=\frac{\partial \tilde{\varphi}}{\partial \tilde{r}}(0)=0 ; \\
& \tilde{q}(0)=e, D(q)(0)=\frac{\partial \tilde{q}}{\partial \tilde{r}}(0)=0 .
\end{aligned}
$$

From Figures $\mathbf{3}$ and $\mathbf{4}$ follow that solutions exist for this case in the domain less than $r / r_{0} \cong 1.1$.

From calculations follow that proton and electron can be considered like charged balls (shortly CB model) which charges are concentrated mainly in the shell of these balls. Relativistic consideration (see also [22]) cannot change this conclusion based on principal of nonlocal physics.

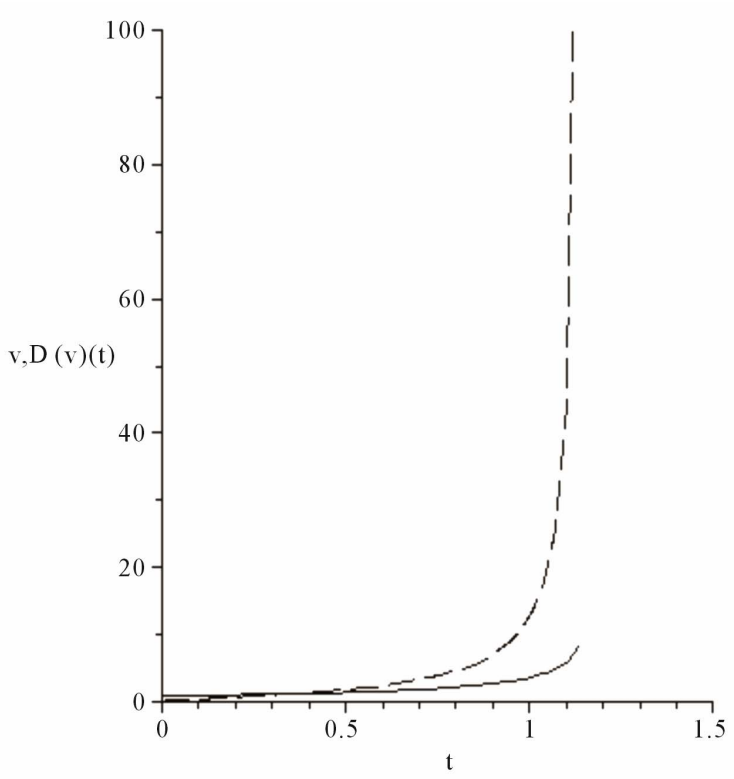

Figure 3. $\mathbf{v}=\tilde{\varphi}(\tilde{\boldsymbol{r}})$, (solid line); $D(\mathbf{v})(t)=\frac{\partial \tilde{\varphi}}{\partial \tilde{\boldsymbol{r}}}(\tilde{\boldsymbol{r}}) \quad$ (dashed line) for electron.

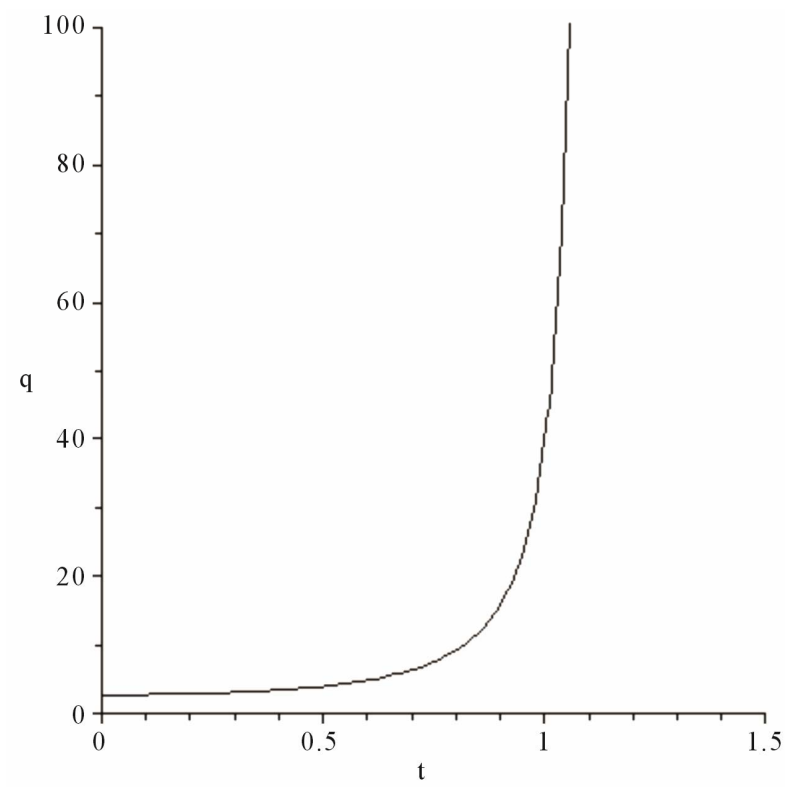

Figure 4. $q=\tilde{\boldsymbol{q}}(\tilde{\boldsymbol{r}})$ for electron.

In the developed theory spin and magnetic moments of proton and electron can be introduced without changing the main conclusions. Really the mentioned characteristics correspond to $\varepsilon_{\alpha}$-internal energy for the particles of $\alpha$-species. For example for electron

$$
\varepsilon_{e l}=\varepsilon_{e l, s p}+\varepsilon_{e l, m},
$$

where $\varepsilon_{e l, s p}=\hbar \omega / 2$, and $\varepsilon_{e l, m}=-\boldsymbol{p}_{\boldsymbol{m}} \cdot \boldsymbol{B}_{\mathrm{int}} ; \boldsymbol{p}_{\boldsymbol{m}}$-electron magnetic moment; $\boldsymbol{B}_{\text {int }}$-intrinsic electron magnetic induction. But

$$
p_{m}=-\frac{e}{m_{e}} \frac{\hbar}{2}, \text { then } \varepsilon_{e l}=\frac{\hbar}{2} \omega_{e f f} .
$$

As it follows from (2.6) written in the spherical coordinate system by the condition $\omega_{\text {eff }}=$ const all previous calculations take place with the additional relation $\frac{5}{2} m_{e} \frac{p}{\rho}+\varepsilon_{e l}=$ const .

\section{To the Theory of Proton and Electron as Ball-Like Charged Objects}

The affirmation that proton and electron can be considered as the ball-like charged objects radically changes the theoretical results of $e^{-} p$ scattering. The wave length $\lambda$ is correlated with the particle impulse $p_{p}$ as $\lambda=h / p_{p}$, this relation leads to condition of the particle localization. At very low electron energies when the wave length is much more than the proton radius $r_{p}$ the scattering is equivalent to that from "point-like" spin-less object. The localization begins when $\lambda \sim r_{p}$ and impulse is about $1 \mathrm{GeV} / \mathrm{c}$. At high electron energies $\lambda<r_{p}$, the wavelengths is sufficiently short to resolve substruc- 
ture.

It is reasonable to write down the Rosenbluth formula for elastic $e^{-} p$ scattering expressed as

$$
\frac{\mathrm{d} \sigma}{\mathrm{d} \Omega}=\left(\frac{\mathrm{d} \sigma}{\mathrm{d} \Omega}\right)_{\text {Mott }}\left(\frac{G_{E}^{2}+\tau_{p} G_{M}^{2}}{1+\tau_{p}}+2 \tau_{p} G_{M}^{2} \tan ^{2} \frac{\theta}{2}\right),
$$

where $\left(\frac{\mathrm{d} \sigma}{\mathrm{d} \Omega}\right)_{\text {Mott }}$ is the Mott differential cross-section which includes the proton recoil. It corresponds to scattering from a spin-0 proton. Formula (4.1) formally is valid for extended charged object. With this aim Rosenbluth introduced the dimensionless electric $G_{E}$ and magnetic $G_{M}$ form factors. Formula (4.1) contains also Lorentz invariant quantity $\tau_{p}=-\frac{q_{p}^{2}}{4 M^{2} c^{2}}>0$. The form factors are a function of $q_{p}^{2}$ rather than scalar production $\left(\boldsymbol{p}_{\boldsymbol{p}}-\boldsymbol{p}_{\boldsymbol{p}}^{\prime}\right)^{2}$ in the three dimensional space and generally speaking cannot be considered in terms of the Fourier transformation of the charge and magnetic moment distribution. If $E$ and $E^{\prime}$ are initial and final electron energies than

$$
q_{p}^{2}=\frac{1}{c^{2}}\left(E-E^{\prime}\right)^{2}-\left(\boldsymbol{p}_{\boldsymbol{p}}-\boldsymbol{p}_{\boldsymbol{p}}^{\prime}\right)^{2}
$$

or

$$
-\left(\boldsymbol{p}_{\boldsymbol{p}}-\boldsymbol{p}_{\boldsymbol{p}}^{\prime}\right)^{2}=q_{p}^{2}\left[1-\left(\frac{q_{p}}{2 M c}\right)^{2}\right]
$$

only if

$$
\frac{\left|q_{p}^{2}\right|}{4 M^{2} c^{2}} \ll 1
$$

one obtains $q_{p}^{2} \approx-\left(\boldsymbol{p}_{\boldsymbol{p}}-\boldsymbol{p}_{\boldsymbol{p}}^{\prime}\right)^{2}$ with typical approximation

$$
G_{E}=G_{E}\left(\left(\boldsymbol{p}_{\boldsymbol{p}}-\boldsymbol{p}_{\boldsymbol{p}}^{\prime}\right)^{2}\right), G_{M}=G_{M}\left(\left(\boldsymbol{p}_{\boldsymbol{p}}-\boldsymbol{p}_{\boldsymbol{p}}^{\prime}\right)^{2}\right) .
$$

In the limit (4.4) the Fourier transforms are used like

$$
\begin{aligned}
G_{E}\left(q_{p}^{2}\right) & =\int \mathrm{d}^{3} \boldsymbol{r}\left\{q(\boldsymbol{r}) \exp \left[\mathrm{i}\left(\tilde{\boldsymbol{p}}-\tilde{\boldsymbol{p}}^{\prime}\right) \cdot \tilde{\boldsymbol{r}}\right]\right\}, \\
G_{M}\left(q_{p}^{2}\right) & =\int \mathrm{d}^{3} \boldsymbol{r}\left\{\mu(\boldsymbol{r}) \exp \left[\mathrm{i}\left(\tilde{\boldsymbol{p}}-\tilde{\boldsymbol{p}}^{\prime}\right) \cdot \tilde{\boldsymbol{r}}\right]\right\} .
\end{aligned}
$$

Rosenbluth formula is derived for a spin-half Dirac particle, then for magnetic moment

$$
\boldsymbol{\mu}=\frac{e}{M} \boldsymbol{S} .
$$

The typical experimental correction leads to additional coefficient

$$
\boldsymbol{\mu}=2.79 \frac{e}{M} \boldsymbol{S}
$$

All calculations depends significantly on the choice of the approximation for the charge density $q(\boldsymbol{r})$. Until now the following $q(\boldsymbol{r})$ approximations are considered: point-like, exponential dependence which grows smaller with the distance from the proton center, Gaussian, uniform sphere, Fermi function. In general the calculations are sensitive to the choice of

$$
\tau_{p}=-\frac{q_{p}^{2}}{4 M^{2} c^{2}}>0 .
$$

For low $\left|q_{p}^{2}\right|\left(\tau_{p} \approx 0\right)$ we have

$$
\frac{\mathrm{d} \sigma}{\mathrm{d} \Omega}=\left(\frac{\mathrm{d} \sigma}{\mathrm{d} \Omega}\right)_{\text {Mott }} G_{E}^{2} .
$$

$$
\begin{aligned}
& \text { For high }\left|q_{p}^{2}\right|\left(\tau_{p} \gg 1\right) \\
& \qquad \frac{\mathrm{d} \sigma}{\mathrm{d} \Omega}=\left(\frac{\mathrm{d} \sigma}{\mathrm{d} \Omega}\right)_{\text {Mott }} G_{M}^{2}\left(1+2 \tau_{p} \tan ^{2} \frac{\theta}{2}\right) .
\end{aligned}
$$

From the first glance it seems that the theory of elastic scattering needs only in recalculation (4.5), (4.6) using new models for the internal proton and electron charge distribution. But situation is more complicated.

The proton-electron collision in the frame of CBmodel should be considered as collision of two resonators.

In this case can be explained a number of character collisional features depending on the initial and final electron energies and the scattering angle. At low $-q_{p}^{2} \approx\left(\boldsymbol{p}_{\boldsymbol{p}}-\boldsymbol{p}_{\boldsymbol{p}}^{\prime}\right)^{2}$ one observes not only the elastic peak but also the resonance curves typical for the excitations in resonators including discrete and continuum spectra. Resonance curves disappear when the cavity cannot serves as resonator. It is the situation which is wellknown from radio physics. For example the usual resonance band corresponds to approximately $8 \mathrm{GeV}$ and the wave length $\sim 1.5 \times 10^{-14} \mathrm{~cm}$, [25]. It leads to the relation $\lambda / r_{p r} \sim 0.1$. This relation is typical for axially symmetric resonators, (see for example [26]). This paper contains calculations for the complex shaped cavity with axial symmetry; the resonance frequency is about $70 \mathrm{GHz}$ with the character length $\sim 35 \mathrm{~mm}$. In the definite sense it is the similar situation; the cavity contains very complicated topology of the electro-magnetic field. Curves of the equal amplitudes of the intensity of electric field create domains in the form of many "islands"-caustic surfaces of electromagnetic field. These "islands" could be the origin of specific features of the electron scattering usually related with partons or quarks. In support of this conjecture can be indicated the results [27]. In [27] M. Popescu realizes the analyses of the cross-sections by collisions $\pi^{+} p$ and $\pi^{-} p$. It was noticed that the experimental data converge to show that the curves giving the total cross-section versus energy are smoothed curves, with large peaks (resonance) for medium energies and a 
slow variation with some waving on the high energy side. Popescu points out that the curves exhibit striking resemblance to scattering curves obtained by electron, neutron or X-ray scattering in liquid and amorphous materials. Starting from this observation Popescu developed a method to extract structural information on the proton internal structure, in the hypothesis that ions are scattered by unknown internal centers when they knock the proton. The result of this analysis was very interesting because the total number of scattering centers was estimated as 20 - 30 with the severe variance with the number of quarks now supposed to be integrated in the proton. It should be added that mentioned number of scattering centers is typical for the quantity of "islands" in resonators for the mentioned conditions.

In this connection another interesting problem is arising. Can be experimentally confirmed the resonator model for the electron? In this case it is reasonable to remind one old Blokhintsev paper published in Physics-Uspekhi as the letter to Editor [28]. He considered the process of the interaction neutrino $v$ and electron $e$ with transformation of electron in $\mu$-meson:

$v+e \rightarrow \mu+v^{\prime}$. In this case the energy density $W$ can be estimated as

$$
W=g^{*} \bar{\psi}_{\mathrm{e}} \psi_{\mu} \bar{\psi}_{\nu} \psi_{v^{\prime}},
$$

where $g^{*}$ is Fermi constant, $\psi_{\mathrm{e}}, \psi_{\mu}, \psi_{\nu}$ are wave functions for electron, $\mu$-meson and neutrino correspondingly. Following I.S. Shapiro, Blokhintsev estimated $g^{*}$ as

$$
g^{*} /(\hbar c)=\Lambda_{0}^{2},
$$

with $\Lambda_{0} \sim 10^{-16} \mathrm{~cm}$. His conclusion consists in affirmation that the strong interaction of electron and neutrino takes place when the wave length $\lambda$ of the neutrino wave packet less than $\Lambda_{0}$.

$$
\lambda<\Lambda_{0} .
$$

The inequality (4.12) can be considered as estimation for revealing of the resonance electron properties. Blohintzev supposes that fulfilling of (4.12) leads to the significant changes in the Compton effect and to other changes in electro-magnetic interaction of electrons. It is possible also to wait for the influence of the resonance electron effects on investigation of hypothetical neutrino oscillations.

\section{Conclusions}

Investigation of the inner charge distribution of the proton and electron in the frame of the non-local quantum hydrodynamics leads to following main results:

1) From calculations follow that proton and electron can be considered like charged balls (shortly CB model) which charges are concentrated mainly in the shell of these balls. In the first approximation this result does not depend on the choice of the non-locality parameter.

The proton-electron collision in the frame of CBmodel should be considered as collision of two resonators. Curves of the equal amplitudes of the intensity of electric field create domains in proton in the form of many "islands" - caustic surfaces of electromagnetic field which can serve as additional scattering centers. It can open new way for explanation a number of character collisional features depending on the initial and final electron energies without consideration partons or quarks as scattering centers.

\section{REFERENCES}

[1] B. V. Alekseev, "Matematicheskaya Kinetika Reagiruyushchikh Gazov," Mathematical Theory of Reacting Gases, Nauka, Moscow, 1982.

[2] B. V. Alexeev, "The Generalized Boltzmann Equation, Generalized Hydrodynamic Equations and Their Applications," Philosophical Transactions of the Royal Society, Vol. 349, 1994, pp. 417-443. doi:10.1098/rsta.1994.0140

[3] B. V. Alexeev, "The Generalized Boltzmann Equation," Physica A, Vol. 216, No. 4, 1995, pp. 459-468. doi:10.1016/0378-4371(95)00044-8

[4] B. V. Alekseev, "Physical Basements of the Generalized Boltzmann Kinetic Theory of Gases," Physics-Uspekhi, Vol. 43, No. 6, 2000, pp. 601-629. doi:10.1070/PU2000v043n06ABEH000694

[5] B. V. Alekseev, "Physical Fundamentals of the Generalized Boltzmann Kinetic Theory of Ionized Gases," Physics-Uspekhi, Vol. 46, No. 2, 2003, pp. 139-167. doi:10.1070/PU2003v046n02ABEH001221

[6] B. V. Alexeev, "Generalized Boltzmann Physical Kinetics," Elsevier, Amsterdam, 2004.

[7] B. V. Alexeev, "Generalized Quantum Hydrodynamics and Principles of Non-Local Physics," Journal of Nanoelectronics and Optoelectronics, Vol. 3, No. 3, 2008, pp. 143158. doi:10.1166/jno.2008.207

[8] B. V. Alexeev, “Application of Generalized Quantum Hydrodynamics in the Theory of Quantum Soliton Evolution," Journal of Nanoelectronics and Optoelectronics, Vol. 3, No. 3, 2008, pp. 316-328.

doi:10.1166/jno.2008.311

[9] L. Boltzmann, "Weitere Studien über das Wärmegleichgewicht unter Gasmolekulen," Sitzungsberichte der Kaiserlichen Akademie der Wissenschaften, Vol. 66, No. 2, 1872, p. 275.

[10] L. Boltzmann, "Vorlesungen über Gastheorie," Verlag von Johann Barth, Leipzig, 1912.

[11] S. Chapman and T. G. Cowling, "The Mathematical Theory of Non-uniform Gases," Cambridge University Press, Cambridge, 1952.

[12] I. O. Hirschfelder, Ch. F. Curtiss and R. B. Bird, "Molecular Theory of Gases and Liquids," John Wiley and sons, Inc., New York, 1954. 
[13] J. S. Bell, "On the Einstein Podolsky Rosen Paradox," Physics, Vol. 1, No. 3, 1964, pp. 195-200.

[14] E. Madelung, "Quantum Theory in Hydrodynamical Form," Zeitschrift fur Physik, Vol. 40, No. 3-4, 1927, pp. 322325. doi:10.1007/BF01400372

[15] L. D. Landau, "Zur Theorie der Energieübertragung. II," Physics of the Soviet Union, Vol. 2, 1932, pp. 46-51.

[16] C. Zener, "Non-Adiabatic Crossing of Energy Levels," Proceedings of the Royal Society of London A, Vol. 137, No. 6, 1932, pp. 696-702.

[17] M. Gell-Mann and F. Low, "Bound States in Quantum Field Theory," Physical Review, Vol. 84, No. 2, 1951, p. 350. doi:10.1103/PhysRev.84.350

[18] M. V. Berry, "Quantal Phase Factors Accompanying Adiabatic Changes," Proceedings of the Royal Society of London A, Vol. 392, No. 1802, 1984, pp. 45-47. doi:10.1098/rspa.1984.0023

[19] B. Simon, "Holonomy, the Quantum Adiabatic Theorem and Berry's Phase," Physical Review Letters, Vol. 51, No. 24, 1983, pp. 2167-2170. doi:10.1103/PhysRevLett.51.2167

[20] B. V. Alexeev, A. I. Abakumov and V. S. Vinogradov, "Mathematical Modeling of Elastic Interactions of Fast Electrons with Atoms and Molecules," Communications on the Applied Mathematics, Computer Centre of the USSR Academy of Sciences, Moscow, 1986.

[21] B. V. Alexeev, "Non-Local Physics. Non-Relativistic
Theory," Lambert Academic Press, 2011.

[22] B. V. Alexeev and I. V. Ovchinnikova, "Non-Local Physics. Relativistic Theory," Lambert Academic Press, 2011.

[23] P. A. M. Dirac, "Quantized Singularities in the Electromagnetic Field," Proceedings of the Royal Society of London A, Vol. 133, No. 821, 1931, pp. 60-72. doi:10.1098/rspa.1931.0130

[24] P. A. M. Dirac, "The Theory of Magnetic Monopoles," Physical Reviews, Vol. 74, No. 7, 1948, pp. 817-830. doi:10.1103/PhysRev.74.817

[25] M. Breidebach, J. I. Friedman, H. W. Kendall, E. D. Bloom, D. H. Coward, H. DeStaebler, J. Drees, L. W. Mo and R. E. Taylor, "Observed Behavior of Highly Inelastic Electron-Proton Scattering," Physical Review Letters, Vol. 23, No. 16, 1969, pp. 935-939. doi:10.1103/PhysRevLett.23.935

[26] A. Yu. Popkov and I. K. Kuzmichev, “Open Resonator with Fragment of Circular Waveguide: Model Computation and Experiment," Radio-Physics and Radio-Astronomy, Vol. 14, No. 4, 2009, pp. 425-432.

[27] M. Popescu, "Proton Internal Structure Revealed by Pion Scattering," Romanian Reports in Physics, Vol. 57, No. 4, 2005, pp. 795-799.

[28] D. I. Blokhintsev, "When the Weak Interaction Becomes the Strong One?" Physics-Uspekhi, Letter to Editor, LXII, Vol. 3, 1957, pp. 381-383. 